\title{
Syntheses, Structures, and Reactions of Heptacoordinate Trihalogermanes Bearing a Triarylmethyl-type Tetradentate Ligand
}

Kohei Iwanaga, ${ }^{\dagger}$ Junji Kobayashi, ${ }^{\dagger}$ Takayuki Kawashima, ${ }^{*}{ }^{\dagger}$ Nozomi Takagi, ${ }^{\ddagger}$ and Shigeru Nagase ${ }^{\ddagger}$

${ }^{\dagger}$ Department of Chemistry, Graduate School of Science, The University of Tokyo, 7-3-1 Hongo, Bunkyo-ku, Tokyo 113-0033, Japan

${ }^{ \pm}$Department of Theoretical Molecular Science, Institute for Molecular Science, Myodaiji, Okazaki 444-8585, Japan

\section{Supporting Information}



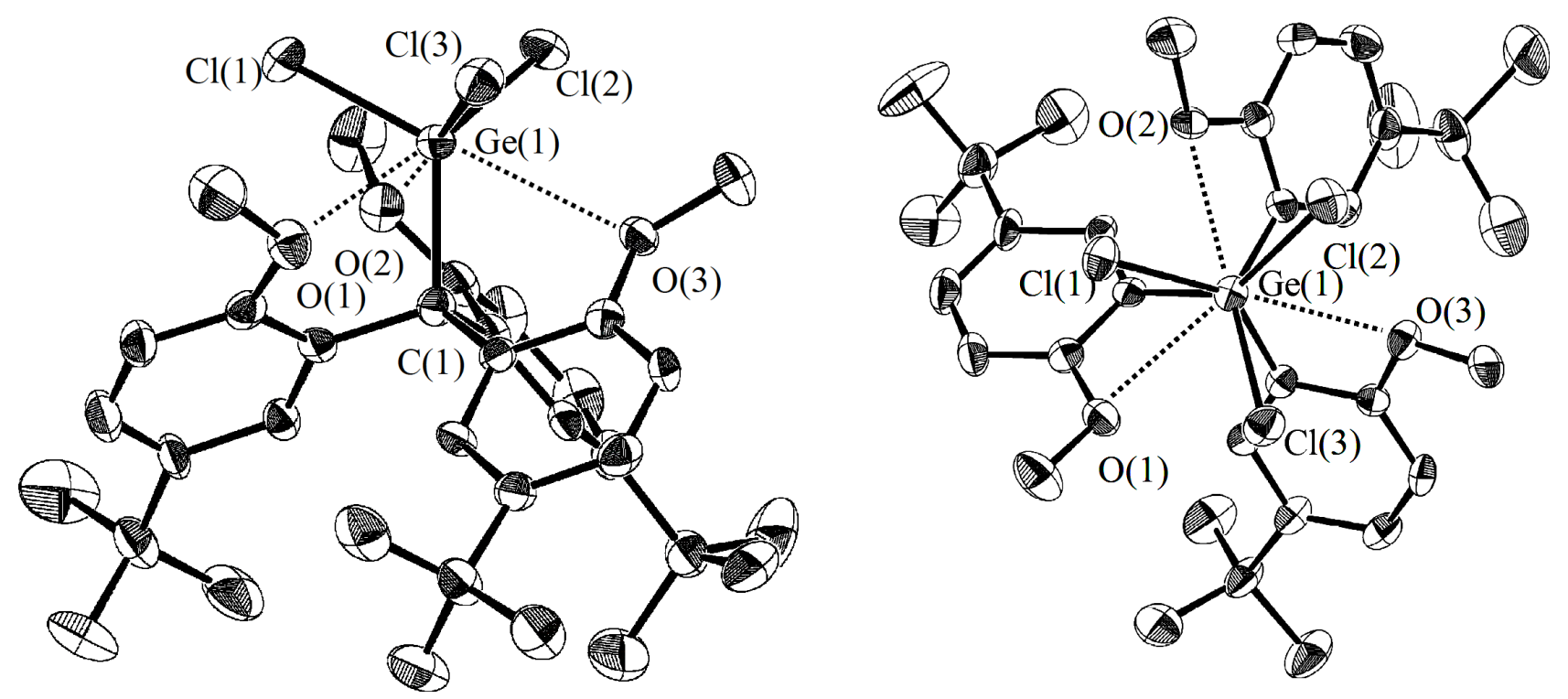

Figure S1. ORTEP drawing of 3 (50\% probability. Left: side view; right: top view).
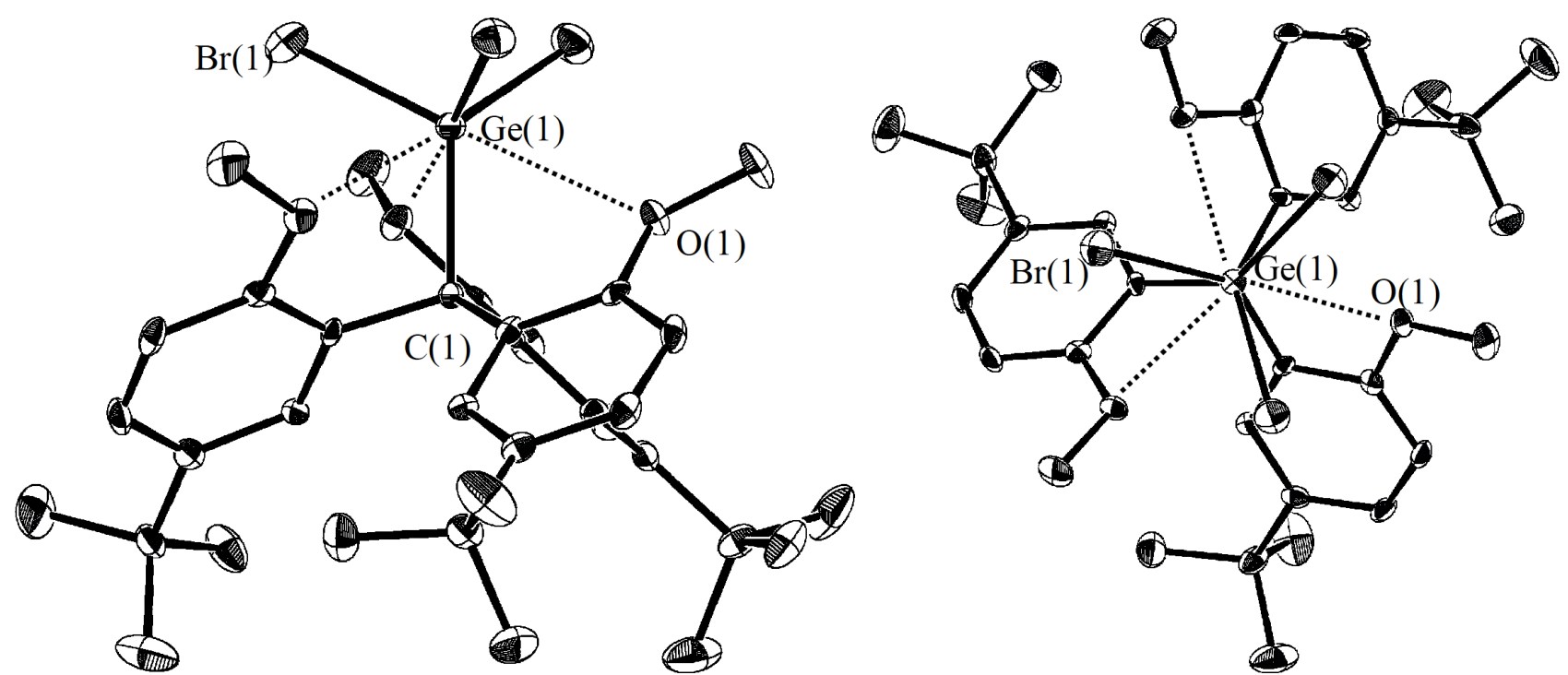

Figure S2. ORTEP drawing of 5 (50\% probability. Left: side view; right: top view). 


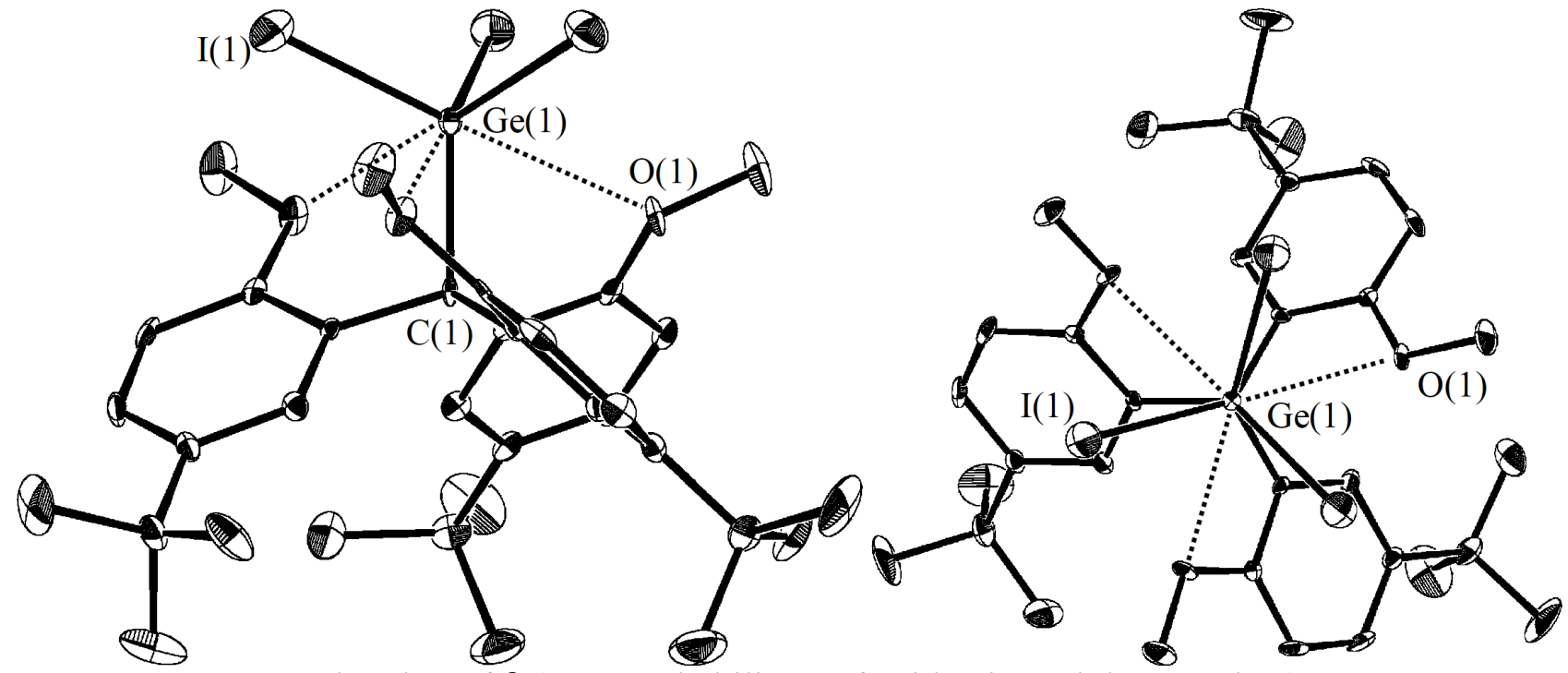

Figure S3. ORTEP drawing of 6 (50\% probability. Left: side view; right: top view). 